\title{
A RECIPROCAL SUM RELATED TO THE RIEMANN $\zeta$-FUNCTION
}

\author{
LiN XiN AND Li XiAOXUE
}

Abstract. This paper, we using the elementary method and several new inequalities to study the computational problem of one kind reciprocal sums related to the Riemann zeta-function at the point $s=4$, and give an explicit computational formula for it.

Mathematics subject classification (2010): 11B83, 11M06.

Keywords and phrases: Riemann zeta-function, inequality, function $[x]$, computational formula, elementary method.

\section{REFERENCES}

[1] E. C. Titchmarsh, The Theory of the Riemann Zeta-Function, Oxford UP, London, 1951, rev. ed., 1986.

[2] A. IVIC, The Riemann zeta-function, Wiley, New York, 1985.

[3] R. P. Fergusson, An application of Stieltjes integration to the power series coefficients of the Riemann zeta-function, Amer. Math. Monthly, 70 (1963), 60-61.

[4] LIN XIN, Some identities related to the Riemann zeta-function, Journal of Inequalities and Applications 2016, 2016: 32.

[5] H. Ohtsuka And S. Nakamura, On the sum of reciprocal Fibonacci numbers, The Fibonacci Quarterly, 46/46 (2008/2009), 153-159.

[6] HAN ZHANG AND ZHENGANG Wu, On the reciprocal sums of the generalized Fibonacci sequences, Advances in Difference Equations 2013, 2013: 377.

[7] ZheFeng Xu AND TINGTINg WANG, The infinite sum of the cubes of reciprocal Fibonacci numbers, Advances in Difference Equations 2013, 2013: 184.

[8] Zhang Wenpeng and Wang Tingting, The infinite sum of reciprocal Pell numbers, Applied Mathematics and Computation 218 (2012), 6164-6167.

[9] ZHENGANG WU AND HAN ZHANG, On the reciprocal sums of higher-order sequences, Advances in Difference Equations 2013, 2013: 189.

[10] Zhengang Wu And Wenpeng Zhang, The sums of the reciprocals of Fibonacci polynomials and Lucas polynomials, Journal of Inequalities and Applications 2012, 2012: 134.

[11] Zhengang Wu And Wenpeng Zhang, Several identities involving the Fibonacci polynomials and Lucas polynomials, Journal of Inequalities and Applications, 2013, 2013: 205.

[12] T. Komatsu, On the nearest integer of the sum of reciprocal Fibonacci numbers, Aportaciones Matematicas Investigacion 20 (2011), 171-184.

[13] T. KOMATSU AND V. LAOHAKOSOL, On the sum of reciprocals of numbers satisfying a recurrence relation of orders, Journal of Integer Seqences 13, Article ID 10.5.8, 2010.

[14] E. Kilic AND T. ARIKAn, More on the infnite sum of reciprocal usual Fibonacci, Pell and higher order recurrences, Applied Mathematics and Computation 219 (2013), 7783-7788. 